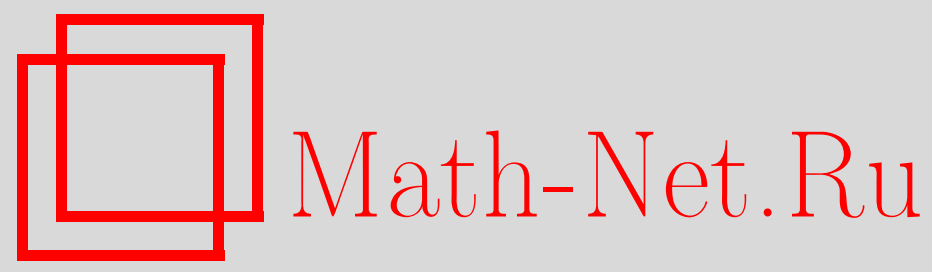

А. В. Ниукканен, Общие линейные преобразования гипергеометрических функций, Матем. заметки, 2001, том 70, выпуск 5, 769-779

DOI: https://doi.org/10.4213/mzm788

Использование Общероссийского математического портала Math-Net.Ru подразумевает, что вы прочитали и согласны с пользовательским соглашением http://www.mathnet.ru/rus/agreement

Параметры загрузки:

IP : 54.237 .206 .68

26 апреля 2023 г., 10:02:06

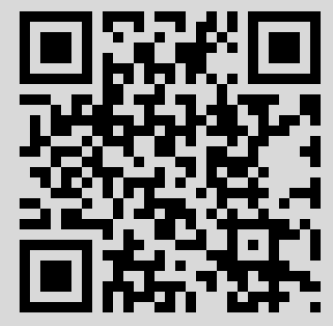




\title{
ОБЩИЕ ЛИНЕЙНЫЕ ПРЕОБРАЗОВАНИЯ ГИПЕРГЕОМЕТРИЧЕСКИХ ФУНКЦИЙ
}

\section{А. В. Ниукканен}

\begin{abstract}
Введено понятие канонической формы гипергеометрического ряда от нескольких переменных. На этой основе с помощью метода факторизации, предложенного в более ранних работах автора, получены явные и наиболее общие формулировки линейных преобразований многократных рядов, имеющих гауссовский, куммеровский или бесселевский тип по некоторому переменному $x_{n}$. Дана полная классификация 34 рядов Горна по типам и формам. С ее помошью показано, что полученные в заметке преобразования позволяют получить 147 однократных преобразований горновских рядов, а также значительное число повторных преобразований. Разработана программа, реализующая полученные преобразования на ЭВМ. Программа выполнена в виде надстройки над системой компьютерной алгебры Maple V-4.

Библиография: 15 названий.
\end{abstract}

1. Введение. Основная цель настоящей заметки состоит в приложении метода операторной факторизации [1]-[4] к исследованию линейных преобразований гипергеометрических рядов от многих переменных. Формулы преобразования, выражающие некоторый ряд в виде другого ряда (или комбинации некоторых рядов), имеют важные применения в разнообразных задачах анализа, теории дифференциальных уравнений, математической физики и квантовой механики. С ростом числа переменных и усложнением формы коэффициентов ряда количество преобразований, вьполняющихся для гипергеометрической функции заданного порядка, быстро увеличивается. Поэтому в рамках традиционных подходов, имющих дело с отдельными функциями, в явном виде удается исследовать “лишь немногие из них" [5].

Как показано в данной заметке, отмеченную трудность удается преодолеть, вводя классификацию гипергеометрических функций по типам и используя каноническую форму записи рядов, объединяющую все функции данного типа. Это позволяет свести исследование любых рядов второго порядка к изучению трех классов функций, имеющих тип $(2 / / 1),(1 / / 1)$ или (0//1) по любому переменному (относительно обозначений и терминологии см. [1]). В случае общих линейных преобразований достаточно исследовать всего лишш две канонические формы типа (2//1) и (1//1), каждая из которых допускает

Работа выполнена при поддержке Российского фонда фундаментальных исследований, грант № 97-01-00317. Продолжение исследований, связанных с дальнейщей разработкой метода факторизации и теории преобразований гипергеометрических функций, также получило поддержку Российского фонда фундаментальных исследований, грант № 01-01-00380. 
явную формулировку соответствующих трансформационных свойств. В результате обширное многообразие общих и частных (обусловленных ограничениями на параметры) линейных преобразований рядов второго порядка от любого числа переменных сводится к однократному или повторному применению четырех nросты ся аналогами стандартных преобразований Куммера и Эйлера [5]. Полезность полученных в работе формул иллюстрируется вьводом некоторьх преобразований функций Горна (случай двух переменных). Разработанная совместно с О. С. Парамоновой программа для ЭВМ способна осуществлять любые линейные преобразования и их комбинации для гипергеометрических рядов от многих переменных.

Ссылки на работы, цитированные в списке литературы, даются в дальнейшем в “двухкомпонентном” виде; например, символ (IV.2) означает ссылку на формулу (2) из работы [4]. В аналогичной форме, но уже без скобок, даются ссылки на определения, замечания и предложения.

2. Канонические формы рядов от многих переменных. Поскольку одно из переменных любого ряда от многих переменных играет в дальнейщем выделенную роль, вместо принятой в [1] записи ${ }^{N} F[L ; \boldsymbol{x}]$ (см. (I.1)) будем использовать для такого ряда “дифференцированное" обозначение ${ }^{N+1} F\left[L_{0}, L^{*} ; x_{0}, \boldsymbol{x}\right]$, где выделенное переменное $x_{0}$, по которому ряд ${ }^{N+1} F \equiv{ }^{N+1} F_{Q}^{P}$ имеет заданный тип $(P / / Q)$ (см. определение I.5), занимает в списке аргументов первую позицию (см. замечание I.4), а совокупность остальных переменных, входящих в любые дальнейшие преобразования однотипньп образом, обозначена векторным символом $\boldsymbol{x}=\left[x_{1}, \ldots, x_{N}\right]$ (относительно векторной нотации см. п. І.2). Для обозначения наборов любых величин, связанных с переменными $x_{0}$ и $\boldsymbol{x}$, также будем использовать раздельные обозначения. Например, элементарные списки параметров, входящие в полньй список $L$ (см. определение I.1), будем записывать в виде $\left\langle\alpha \mid m_{0}, \boldsymbol{m}\right\rangle,\left\langle\beta \mid l_{0}, \boldsymbol{l}\right\rangle$ и т.п. В список $L_{0}$ будем включать такие элементарные списки $\left\langle\alpha \mid m_{0}, \boldsymbol{m}\right\rangle$, для которьх $m_{0} \neq 0$. Элементарные списки вида $\langle\alpha \mid 0, \boldsymbol{m}\rangle$ будем включать в список $L^{*}$. Из определения I.1 следует, что соответствующие списку $L^{*}$ коэффициенты $L\left(i_{1}, \ldots, i_{N}\right)$ не зависят от переменного суммирования $i_{0}$, связанного с аргументом $x_{0}$.

ОПРЕДЕЛЕНИЕ 1. Канонической формой $p_{я д а ~}{ }^{N+1} F\left[L_{0}, L^{*} ; x_{0}, \boldsymbol{x}\right]$ по переменному $x_{0}$ будем называть такую форму записи этого ряда, для которой спектральные индексы, связанные с переменным $x_{0}$, имеют неотрицательные значения.

В большинстве случаях определение подразумевает дополнительное преобразование таких индексов к единичным значениям. Возможность записи любого гипергеометрического ряда ${ }^{N+1} F$ в канонической форме по любому переменному следует из замечания I.1в. Из определения I.5 следует, что куммеровская и гауссовская канонические формы даются выражениями

$$
\begin{aligned}
& \mathscr{K}={ }^{N+1} F_{1}^{1}\left(x_{0}, \boldsymbol{x}\right)={ }^{N+1} F\left[\left[\left\langle\nu_{1} \mid 1, \boldsymbol{m}_{1}\right\rangle / /\left\langle\nu_{0} \mid 1, \boldsymbol{m}_{0}\right\rangle\right], L^{*} ; x_{0}, \boldsymbol{x}\right], \\
& \mathscr{G}={ }^{N+1} F_{1}^{2}\left(x_{0}, \boldsymbol{x}\right)={ }^{N+1} F\left[\left[\left\langle\nu_{1} \mid 1, \boldsymbol{m}_{1}\right\rangle,\left\langle\nu_{2} \mid 1, \boldsymbol{m}_{2}\right\rangle / /\left\langle\nu_{0} \mid 1, \boldsymbol{m}_{0}\right\rangle\right], L^{*} ; x_{0}, \boldsymbol{x}\right] .
\end{aligned}
$$

В общем случае ряды ${ }^{N+1} F_{1}^{1}$ и ${ }^{N+1} F_{1}^{2}$ могут иметь любой порядок, так как определения (1) и (2) не накладывают никаких ограничений на структуру списка $L^{*}$. Множество таких форм наряду с выражениями (1), (2) содержат каноническую форму $\mathscr{B}={ }^{N+1} F_{1}^{0}$ бесселевского типа $(0 / / 1)$, соответствующую списку $L_{0}=\left[* / /\left\langle\nu_{0} \mid 1, \boldsymbol{m}_{0}\right\rangle\right]$, а также специальную каноническую форму гауссовского типа $\mathscr{G}^{\prime}={ }^{N+1} F_{1}^{2}$, соответствующую 
списку $L_{0}=\langle\nu \mid 2, \boldsymbol{m}\rangle / /\left\langle\nu_{0} \mid 1, \boldsymbol{m}_{0}\right\rangle$. Для вывода общих линейных преобразований достаточно ограничиться формами $\mathscr{G}$ и $\mathscr{K}$, так как для формы $\mathscr{B}$ линейные преобразования вообще не имеют места, а те линейные преобразования, которые выполняются для формы $\mathscr{G}^{\prime}$, являются частными случаями соответствующих преобразований формы $\mathscr{G}$ (анализ квадратичных преобразований форм $\mathscr{B}$ и $\mathscr{G}$ в задачу данной заметки не входит).

\section{3. Соотношения $\Omega$-эквивалентности.}

ОПРЕДЕЛЕНИЕ 2. Функциональное соотношение $f_{1}(x)=f_{2}(x)$ назовем $\Omega$-әквивалентным соотношению $F_{1}(x)=F_{2}(x)$, если найдется такая функция $\Psi(x)$, что

$$
f_{1}(x)=\left.\Psi(d(s)) F_{1}(x s)\right|_{s=0}, \quad f_{2}(x)=\left.\Psi(d(s)) F_{2}(x s)\right|_{s=0} .
$$

ОПРЕДЕЛЕНИЕ 3. Назовем операторы $F_{1}(d(s), s), F_{2}(d(s), s)$ $\Omega$-эквивалентныли друг другу, если

$$
\left.F_{1}(d(s), s) \Psi(x s)\right|_{s=0}=\left.F_{2}(d(s), s) \Psi(x s)\right|_{s=0}
$$

для любой $\Psi(x)$. Соотношение $\Omega$-эквивалентности (4) будем записьвать для краткости в виде $F_{1} \Leftrightarrow F_{2}$. Формулы $(3),(4)$ относятся к числу соотношений, образуюших кониептуальную основу метода факторизачии.

Отметим, что из операторного соотношения $F_{1} \Leftrightarrow F_{2}$, вообще говоря, не следует функциональное соотношение $F_{1}=F_{2}$, так как операторы $F_{1}$ и $F_{2}$ могут зависеть не только от операторов дифференцирования $d(s)$, но и от переменных дифференцирования $s$. Например, с помощью формулы смешения операторного аргумента

$$
\exp (-z s) F[d(s)] \exp (z s)=F[d(s)+z]
$$

и формулы униформизации аргумента биномиального ряда

$$
F_{0}^{1}[\nu ; x+z]=(1-x-z)^{-\nu}=(1-z)^{-\nu} F_{0}^{1}\left[\nu ;(1-z)^{-1} x\right]
$$

получаем соотношения $\Omega$-эквивалентности

$$
\begin{aligned}
F[d(s)] \exp (z s) & \Leftrightarrow F[d(s)+z], \\
F_{0}^{1}[\nu ; d(s)] \exp (z s) & \Leftrightarrow(1-z)^{-\nu} F_{0}^{1}\left[\nu ;(1-z)^{-1} d(s)\right],
\end{aligned}
$$

однако никаких простых функциональных соотношений, связанных с этими операторными тождествами, не существует. Формулы (5)-(7) дают типичные примеры соотношений, составляющих техническую основу метода.

В силу определения 2 любое функциональное соотношение $F_{1}=F_{2}$ порождает некоторьй класс $\Omega$-эквивалентных соотношений вида $\Psi * F_{1}=\Psi * F_{2}$, где

$$
\left.\langle\Psi * F \mid x\rangle \equiv \Psi(d(s)) F(x s)\right|_{s=0}
$$

- $\Omega$-произведение функций $\Psi, F$. 
ЗАмЕЧАниЕ 1. Для доказательства соотношения, принадлежащего некоторому классу $\Omega$-эквивалентных формул, достаточно доказать любое соотношение этого класса.

ОПРЕДЕЛЕниЕ 4. Простейшее по форме соотношение, принадлежащее некоторому классу $\Omega$-эквивалентных формул, будем называть протосоотношением, порождающим данное семейство формул.

Для применения метода $\Omega$-эквивалентных соотношений к анализу трансформационных свойств рядов (1) и (2) введем ряд

$$
b={ }^{N+1} F_{0}^{1}\left(x_{0}, \boldsymbol{x}\right)={ }^{N+1} F\left[\langle\nu \mid 1, \boldsymbol{m}\rangle, L^{*} ; x_{0}, \boldsymbol{x}\right],
$$

имеющий биномиальньй тип $(1 / / 0)$ по переменному $x_{0}$, и получим для него некоторое преобразование, играющее роль протосоотношения по отношению к семейству общих линейных преобразований рядов от многих переменных, отыскание которых составляет основную цель данной заметки.

ЗАмЕчАниЕ 2 (элементарная редукция биномиального типа). Гипергеометрический ряд $b \equiv{ }^{N+1} F_{0}^{1}\left(x_{0}, \boldsymbol{x}\right)$ (см. (8)), имеющий биномиальньй тип $(1 / / 0)$ по переменному $x_{0}$, при любых значениях параметров выражается в виде гипергеометрического ряда от $N$ переменных.

ДокАЗАТЕЛЬСтво. Применяя к ряду $b$ обобщенную формулу факторизации (I.8), имеем $b=\left.F_{0}^{1}[\nu ; d(s)] \exp \left(x_{0} s\right)^{N} F\left[L^{*} ; \boldsymbol{x} s^{\boldsymbol{m}}\right]\right|_{s=0}$. Используя соотношение $\Omega$-эквивалентности (7) и вновь применяя формулу (I.8), получаем

$$
b \equiv{ }^{N+1} F\left[\langle\nu \mid 1, \boldsymbol{m}\rangle, L^{*} ; x_{0}, \boldsymbol{x}\right]=\left(1-x_{0}\right)^{-\nu N} F\left[\langle\nu \mid \boldsymbol{m}\rangle, L^{*} ;\left(1-x_{0}\right)^{-\boldsymbol{m}} \boldsymbol{x}\right],
$$

что завершает доказательство замечания 2 и одновременно дает его явную формулировку.

ЗАмЕчАнИЕ 3 . Для ряда $b \equiv{ }^{N+1} F_{0}^{1}\left(x_{0}, \boldsymbol{x}\right)$, имеющего биномиальньй тип $(1 / / 0)$ по переменному $x_{0}$, выполняется соотношение инвариантности

$$
\begin{aligned}
& \left(1-x_{0}\right)^{\nu N+1} F\left[\langle\nu \mid 1, \boldsymbol{m}\rangle, L^{*} / /\langle\nu \mid 0, \boldsymbol{m}\rangle ; x_{0},\left(1-x_{0}\right)^{\boldsymbol{m}} \boldsymbol{x}\right] \\
& \quad=\left(1-x_{0}^{\prime}\right)^{\nu^{\prime} N+1} F\left[\left\langle\nu^{\prime} \mid 1, \boldsymbol{m}^{\prime}\right\rangle, L^{*} / /\left\langle\nu^{\prime} \mid 0, \boldsymbol{m}^{\prime}\right\rangle ; x_{0}^{\prime},\left(1-x_{0}^{\prime}\right)^{\boldsymbol{m}^{\prime}} \boldsymbol{x}\right] .
\end{aligned}
$$

ДокАЗАТЕльство замечания 3 следует из формулы (9), с помошью которой обе части (10) выражаются в виде ряда ${ }^{N} F[L ; \boldsymbol{x}]$, не зависящего от параметров $x_{0}, \nu, \boldsymbol{m}$.

В частном случае $x_{0}^{\prime}=x_{0} /\left(x_{0}-1\right)$ формула (10) с помощью замен $L^{*} \rightarrow\left[L^{*},\langle\nu \mid 0, \boldsymbol{m}\rangle\right]$, $\boldsymbol{x} \rightarrow\left(1-x_{0}\right)^{-\boldsymbol{m}} \boldsymbol{x}$ и переобозначений $\nu=\nu_{1}, \nu^{\prime}=\nu_{0}-\nu_{1} \equiv \nu_{0 \overline{1}}, \boldsymbol{m}^{\prime}=\boldsymbol{m}_{0}-\boldsymbol{m}_{1} \equiv \boldsymbol{m}_{0 \overline{1}}$ получает вид

$$
\begin{aligned}
& F\left[\left\langle\nu_{1} \mid 1, \boldsymbol{m}_{1}\right\rangle, L^{*} ; x_{0}, \boldsymbol{x}\right] \\
& =\left(1-x_{0}\right)^{-\nu_{0}} F\left[\begin{array}{l}
\left.\left\langle\nu_{0 \overline{1}} \mid 1, \boldsymbol{m}_{0 \overline{1}}\right\rangle,\left\langle\nu_{1} \mid 0, \boldsymbol{m}_{1}\right\rangle, L^{*} ; \frac{x_{0}}{x_{0}-1}, \frac{\boldsymbol{x}}{\left(1-x_{0}\right)^{\boldsymbol{m}_{0}}}\right] . \\
\left\langle\nu_{0 \overline{1}} \mid 0, \boldsymbol{m}_{0 \overline{1}}\right\rangle
\end{array}\right.
\end{aligned}
$$

Формула (11) является протосоотношением, порождающим искомое семейство общих линейных преобразований. 
4. Элементарные правила редукции. Вспомогательные преобразования. Прежде чем перейти к формулировке общих линейных преобразований, перечислим некоторые простые утверждения и вспомогательные приемы, значительно облегчающие последующий анализ линейных преобразований и их многочисленных следствий, к числу которых относятся в первую очередь формулы приведения многократных гипергеометрических рядов.

ЗАмЕчАнИЕ 4 . К числу элементарных приемов, играющих наряду с формулой (9) важную вспомогательную роль в преобразованиях гипергеометрических рядов, относятся:

(a) сокращение или, напротив, нарашивание одинаковых параметров в числителе и знаменателе списка $L$;

(б) “растягивание” или “стягивание" ряда ${ }^{N} F$ по числу переменных;

(в) приведение ряда ${ }^{N} F$ специального вида к формепроизведения рядов от меньшего числа переменных или, напротив, формальная запись произведения нескольких рядов в виде одного гипергеометрического ряда;

(г) уменьшение числа переменных ряда благодаря обращению в нуль параметра числителя, характеризуемого неотрищательными спектральными числами.

Кратко поясним смысл отдельных приемов, упомянутых в замечании 4.

ЗАмЕчАниЕ 4a (элементарная редукция типа сокращения параметров). Смысл замечания 4а состоит в том, что сокращение параметров приводит к упрощению формальной структуры ряда, а введение добавочных параметров с помощюю последующего применения формул факторизации позволяет отнести эти параметры к разным рядам и осушествить над ними разные преобразования, что иногда приводит к полезным нетривиальньм следствиям.

ЗАмЕчАниЕ 4 (элементарная редукция типа "стягивания" переменных). Смысл замечания $4 б$ состоит в том, что если на некотором множестве индексов, нумерующих члены спектральных наборов, компоненты спектрального набора любого параметра ряда ${ }^{N} F$ принимают одно и то же значение (не обязательно одинаковое для разных наборов), то соответствующий ряд “стягивается” к ряду от меньшего числа переменных. Напротив, с помощью формального обращения этого правила любой ряд можно "растянуть" по числу переменных. Примеры:

$$
\begin{aligned}
& { }^{5} F\left[\left\langle\boldsymbol{d}_{1} \mid 12333\right\rangle,\left\langle\boldsymbol{d}_{2} \mid 21111\right\rangle,\left\langle\boldsymbol{d}_{3} \mid 13222\right\rangle ; x_{1}, x_{2}, x_{3}, x_{4}, x_{5}\right] \\
& ={ }^{3} F\left[\left\langle\boldsymbol{d}_{1} \mid 123\right\rangle,\left\langle\boldsymbol{d}_{2} \mid 211\right\rangle,\left\langle\boldsymbol{d}_{3} \mid 132\right\rangle ; x_{1}, x_{2}, x_{3}+x_{4}+x_{5}\right], \\
& F_{1}^{0}\left[* / / \alpha ; x_{1}+x_{2}\right]={ }^{2} F\left[* / / \alpha: * ; * ; x_{1}, x_{2}\right]
\end{aligned}
$$

и т.п.

Доказательство соотношений (12), (13) сводится к использованию формул факторизации с последующим применением формулы сложения для показательных функций. Для пояснения замечания 4в введем

ОПРЕДЕЛЕНИЕ 5. Множество номеров $i$, для которых $m_{i} \neq 0(i=1,2, \ldots, N)$, назовем носителем спектрального набора $\boldsymbol{m}$. Объединение носителей совокупности нескольких спектров будем назьвать носителем данной группы спектров. 
ЗАмЕчАниЕ 4в (элементарная редукция типа факторизации). Смысл замечания 4в состоит в том, что если спектры, входящие в список $L$, можно разбить на групш с непересекающимися носителями, то ряд ${ }^{N} F$ разбивается в произведение рядов, соответстующих этим групшам. Например,

$$
\begin{aligned}
& { }^{5} F\left[\left\langle\boldsymbol{d}_{1} \mid m_{1} m_{2} m_{3} 00\right\rangle,\left\langle\boldsymbol{d}_{2} \mid l_{1} l_{2} l_{3} 00\right\rangle,\left\langle\boldsymbol{d}_{3} \mid 000 p_{1} p_{2}\right\rangle ; x_{1}, x_{2}, x_{3}, x_{4}, x_{5}\right] \\
& \quad={ }^{3} F\left[\left\langle\boldsymbol{d}_{1} \mid m_{1} m_{2} m_{3}\right\rangle,\left\langle\boldsymbol{d}_{2} \mid l_{1} l_{2} l_{3}\right\rangle ; x_{1}, x_{2}, x_{3}\right] \cdot{ }^{2} F\left[\left\langle\boldsymbol{d}_{3} \mid p_{1} p_{2}\right\rangle ; x_{4}, x_{5}\right] .
\end{aligned}
$$

ЗАмЕчАниЕ $4 г$ (элементарная редукция типа подавления переменных и параметров). Смысл замечания 4 г состоит в том, что часть переменных суммирования ряда ${ }^{N} F$, соответствующая положительным спектральным числам в выражениях вида $\langle 0| m_{1}, 0$, $\left.m_{2}, m_{3}, 0, \ldots\right\rangle$, обращается в нуль благодаря элементарному правилу отбора для символа Похгаммера. Этоприводит к подавлению всех спектральных компонент и аргументов ряда, связанных с нулевыми переменньми суммирования.

Пример менее тривиального вспомогательного приема, играющего важную роль в преобразованиях гипергеометрических функций от многих переменных, дает

ПРЕдЛОЖЕНИЕ 1 (вспомогательные преобразования рядов, содержащих параметры $\langle 0 \mid 1, \overline{1}\rangle,\langle 0 \mid \overline{1}, \boldsymbol{p}\rangle)$. Гипергеометрический ряд ${ }^{2} F$, содержсащий составной параметр $\langle 0 \mid 1, \overline{1}\rangle$, допускает билинейное преобразование вида

$$
\begin{aligned}
& { }^{2} F\left[\langle 0 \mid 1, \overline{1}\rangle,\left\langle\boldsymbol{d}_{1}^{\prime} \mid m_{1}, m_{2}\right\rangle,\left\langle\boldsymbol{d}_{2}^{\prime} \mid l_{1}, l_{2}\right\rangle, \ldots: \boldsymbol{d}_{1} ; \boldsymbol{d}_{2} ; z_{1}, z_{2}\right] \\
& \quad={ }^{2} F\left[\left\langle\boldsymbol{d}_{2} / / 1 \mid 1,1\right\rangle,\left\langle\boldsymbol{d}_{1}^{\prime} \mid m_{12}, m_{2}\right\rangle,\left\langle\boldsymbol{d}_{2}^{\prime} \mid l_{12}, l_{2}\right\rangle, \ldots: \boldsymbol{d}_{1} ; * ; z_{1} z_{2},-z_{2}\right],
\end{aligned}
$$

где $m_{12}=m_{1}+m_{2}, l_{12}=l_{1}+l_{2}$. Ряд ${ }^{N} F$, содержащий составной параметр $\langle 0 \mid \overline{1}, \boldsymbol{p}\rangle$, $\boldsymbol{p} \equiv\left[p_{1}, \ldots, p_{N}\right]$, әде $p_{1} \geqslant 0, \ldots, p_{N-1} \geqslant 0$, допускает нелинейное преобразование вида

$$
\begin{aligned}
{ }^{N+1} F[ & \left.\langle 0 \mid \overline{1}, \boldsymbol{p}\rangle,\left\langle\boldsymbol{d}_{1}^{\prime} \mid m_{0}, \boldsymbol{m}\right\rangle,\left\langle\boldsymbol{d}_{2}^{\prime} \mid l_{0}, \boldsymbol{l}\right\rangle, \ldots: \boldsymbol{d}_{0} ; \boldsymbol{d}_{1} ; \ldots ; \boldsymbol{d}_{N} ; x_{0}, \boldsymbol{x}\right] \\
= & { }^{N+1} F\left[\left\langle\boldsymbol{d}_{0} / / 1 \mid 1, \boldsymbol{p}\right\rangle,\left\langle\boldsymbol{d}_{1}^{\prime} \mid m_{0}, \boldsymbol{m}+m_{0} \boldsymbol{p}\right\rangle,\left\langle\boldsymbol{d}_{2}^{\prime} \mid l_{0}, \boldsymbol{l}+l_{0} \boldsymbol{p}\right\rangle, \ldots:\right. \\
& \left.* ; \boldsymbol{d}_{1} ; \boldsymbol{d}_{2} ; \ldots ; \boldsymbol{d}_{N} ;-x_{0}, x_{1} x_{0}^{p_{1}}, \ldots, x_{N} x_{0}^{p_{N}}\right] .
\end{aligned}
$$

ДокАЗАтЕльСтво. Если $L^{*}$-пустой набор параметров, тов случае $N=1, m=-1$, $x_{0}=z_{1}, x=z_{2}$ тождество (9) принимает вид

$$
{ }^{2} F\left[\langle\nu \mid 1, \overline{1}\rangle: * ; * ; z_{1}, z_{2}\right]=\left(1-z_{1}\right)^{-\nu} F\left[\langle\nu \mid \overline{1}\rangle ; z_{2}-z_{1} z_{2}\right],
$$

где $\overline{1} \equiv-1$. С помощью замечаний I.1а и 4 б имеем

$$
{ }^{2} F\left[\langle\nu \mid 1, \overline{1}\rangle: * ; * ; z_{1}, z_{2}\right]=\left(1-z_{1}\right)^{-\nu 2} F\left[* / / 1-\nu: * ; * ; z_{1} z_{2},-z_{2}\right] .
$$

В случае $\nu=0$ получаем из (16) преобразование вида

$$
{ }^{2} F\left[\langle 0 \mid 1, \overline{1}\rangle: * ; * ; z_{1}, z_{2}\right]={ }^{2} F\left[* / / 1: * ; * ; z_{1} z_{2},-z_{2}\right] .
$$

Применяя формулу факторизации (см. (I.8)), легко убедиться в том, что соотношение (14) $\Omega$-эквивалентно формуле (17). В силу замечания 1 это наблюдение доказьвает справедливость формулы (14).

Формула (15), являюшаяся прямым обобщением простейшего соотношения (14), может быть доказана либо с помощью метода факторизации по аналогии с доказательством формулы (14), либо заменой переменного суммирования $j_{0}=i_{0}-p_{1} i_{1}-\cdots-p_{N} i_{N}$ в исходном ряде. 
5. Общие линейные преобразования. Линейное преобразование куммеровского типа по переменному $x_{n}$ будем обозначать символом $L^{n}$.

ПРЕДЛОЖЕНИЕ 2. Для ряда $F \equiv{ }^{N+1} F_{1}^{1}\left(x_{0}, \boldsymbol{x}\right)$, имеющего куммеровский тип $(1 / / 1)$ по переменному $x_{0}$, выполняется линейное преобразование $F=L^{0} F$ вида

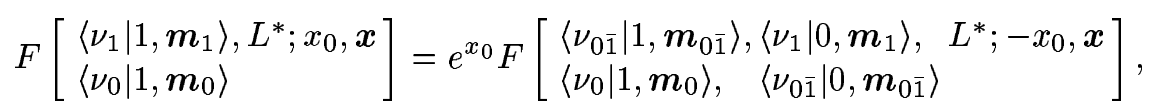

являющееся аналогом преобразования Куммера ряда $F_{1}^{1}(x)$ [5].

Линейные преобразования эйлеровского типа по переменному $x_{n}$, связанные с первым и вторым параметрами числителя ряда $F$, записанного в канонической форме по переменному $x_{n}$, будем обозначать символами $L_{1}^{n}$ и $L_{2}^{n}$ соответственно. Произведение преобразований $L_{1}^{n}, L_{2}^{n}$ обозначим символом $L_{0}^{n}$.

ПРЕДЛОЖЕНИЕ 3. Для ряда $F \equiv{ }^{N+1} F_{1}^{2}\left(x_{0}, x\right)$, имеющего гауссовский тип $(2 / / 1)$ по переменному $x_{0}$, выполняются линейные преобразования

$$
\begin{aligned}
& F\left[\begin{array}{l}
\left\langle\nu_{1} \mid 1, \boldsymbol{m}_{1}\right\rangle,\left\langle\nu_{2} \mid 1, \boldsymbol{m}_{2}\right\rangle, L^{*} ; x_{0}, \boldsymbol{x} \\
\left\langle\nu_{0} \mid 1, \boldsymbol{m}_{0}\right\rangle
\end{array}\right] \\
& =L_{1}^{0} F \equiv\left(1-x_{0}\right)^{-\nu_{2}} F\left[\begin{array}{cc}
\left\langle\nu_{0 \overline{1}} \mid 1, \boldsymbol{m}_{0 \overline{1}}\right\rangle,\left\langle\nu_{2} \mid 1, \boldsymbol{m}_{2}\right\rangle,\left\langle\nu_{1} \mid 0, \boldsymbol{m}_{1}\right\rangle, L^{*} ; \\
\frac{x_{0}}{x_{0}-1}, \frac{\boldsymbol{x}}{\left(1-x_{0}\right)^{\boldsymbol{m}_{2}}} \\
\left\langle\nu_{0} \mid 1, \boldsymbol{m}_{0}\right\rangle,\left\langle\nu_{0 \overline{1}} \mid 0, \boldsymbol{m}_{0 \overline{1}}\right\rangle
\end{array}\right] \\
& =L_{2}^{0} F \equiv\left(1-x_{0}\right)^{-\nu_{1}} F\left[\begin{array}{cc}
\left\langle\nu_{1} \mid 1, \boldsymbol{m}_{1}\right\rangle,\left\langle\nu_{0} \mid 1, \boldsymbol{m}_{0 \overline{2}}\right\rangle, & \left\langle\nu_{2} \mid 0, \boldsymbol{m}_{2}\right\rangle, L^{*} ; \\
\left\langle\nu_{0} \mid 1, \boldsymbol{m}_{0}\right\rangle,\left\langle\nu_{0 \overline{2}} \mid 0, \boldsymbol{m}_{0 \overline{2}}\right\rangle & \frac{x_{0}}{x_{0}-1}, \frac{\boldsymbol{x}}{\left(1-x_{0}\right)^{\boldsymbol{m}_{1}}}
\end{array}\right] \\
& =L_{0}^{0} F \equiv\left(1-x_{0}\right)^{\nu_{0 \overline{1}}} F\left[\begin{array}{rr}
\left\langle\nu_{0 \overline{1}} \mid 1, \boldsymbol{m}_{0 \overline{1}}\right\rangle,\left\langle\nu_{0 \overline{2}} \mid 1, \boldsymbol{m}_{0 \overline{2}}\right\rangle,\left\langle\nu_{1} \mid 0, \boldsymbol{m}_{1}\right\rangle, & \left\langle\nu_{2} \mid 0, \boldsymbol{m}_{2}\right\rangle, L^{*} ; \\
\left\langle\nu_{0} \mid 1, \boldsymbol{m}_{0}\right\rangle,\left\langle\nu_{0 \overline{1}} \mid 0, \boldsymbol{m}_{0 \overline{1}}\right\rangle,\left\langle\nu_{0 \overline{2}} \mid 0, \boldsymbol{m}_{0 \overline{2}}\right\rangle & x_{0}, \frac{\boldsymbol{x}}{\left(1-x_{0}\right)^{\boldsymbol{m}_{\overline{0} 12}}}
\end{array}\right] \text {, }
\end{aligned}
$$

являющиеся аналогами преобразований Эйлера ряда $F_{1}^{2}(x)$ [5].

ДокАЗАТЕЛЬСтво. Соотношения $(20),(21)$ являются прямыми следствиями формулы (19). Поэтому для доказательства предложений 2 и 3 достаточно доказать формулы (18) и (19). Произведя в (18) замены $x_{0} \rightarrow x_{0} s, \boldsymbol{x} \rightarrow \boldsymbol{x} s^{\boldsymbol{m}_{2}}$, применяя к обеим частям оператор $\left.F_{0}^{1}\left[\nu_{2} ; d(s)\right]\right|_{s=0}$, учитьвая соотношение (7) и используя затем формулу (I.8), можно показать, что соотношения (18) и (19) $\Omega$-эквивалентны друг другу. Точно такие же преобразования в случае $\nu_{2}=\nu_{0}, \boldsymbol{m}_{2}=\boldsymbol{m}_{0}$ доказьвают $\Omega$-эквивалентность формул (18) и (11). Применение замечания 1 завершает доказательство предложений 2 и 3 , а из определения 4 следует, что формула (11) является протосоотношением, порождающим семейство $\Omega$-эквивалентньх формул (18)-(20). 
6. Горновские функции. Полная классификация всех 34 горновских функций [5] по типам (см. определение I.6) и каноническим формам (см. п. 2) дает таблища 1, где в квадратных скобках указаны типы рядов, расположенных далее внутри фигурных скобок; буквенные символы внутри круглых скобок (см. п. 2) задают канонические формы по первому и второму аргументу для каждого из горновских рядов, помешенных вслед за круглыми скобками. Вырожденные ряды $\overline{\mathrm{H}}_{1}, \ldots, \overline{\mathrm{H}}_{11}$, обозначаемые в [5] прямым шрифтом, снабжены в таблище дополнительной верхней чертой во избежание путаницы с полньми рядами $H_{1}, \ldots, H_{7}$, обозначаемыми в [5] сходными по начертанию наклонными буквами.

\section{ТАБЛИЦА 1}

$$
\begin{aligned}
& {[2 / / 1,2 / / 1] \quad }\left((\mathscr{G}, \mathscr{G}): F_{1}, F_{2}, F_{3}, F_{4}, G_{1}, G_{2}, H_{1}, H_{2} ;\right. \\
&\left(\mathscr{G}^{\prime}, \mathscr{G}\right): H_{3}, H_{4}, H_{5}, H_{6}, H_{7} ; \\
&\left.\left(\mathscr{G}^{\prime}, \mathscr{G}^{\prime}\right): G_{3}\right\} \\
& {[2 / / 1,1 / / 1] \quad\left\{(\mathscr{G}, \mathscr{K}): \Phi_{1}, \Psi_{1}, \Xi_{1}, \Gamma_{1}, \overline{\mathrm{H}}_{1}, \overline{\mathrm{H}}_{2} ;\right.}\left.\left(\mathscr{G}^{\prime}, \mathscr{K}\right): \overline{\mathrm{H}}_{6}, \overline{\mathrm{H}}_{7}, \overline{\mathrm{H}}_{8}, \overline{\mathrm{H}}_{9}\right\} \\
& {[1 / / 1,2 / / 1] \quad\left\{(\mathscr{K}, \mathscr{G}): \overline{\mathrm{H}}_{11}\right\} } \\
& {[2 / / 1,0 / / 1] \quad\left\{(\mathscr{G}, \mathscr{B}): \Xi_{2}, \overline{\mathrm{H}}_{3} ;\right.} \\
&\left.(\mathscr{G}, \mathscr{B}): \overline{\mathrm{H}}_{10}\right\} \\
& {[1 / / 1,1 / / 1] \quad\left\{(\mathscr{K}, \mathscr{K}): \Phi_{2}, \Psi_{2}, \Gamma_{2}, \overline{\mathrm{H}}_{4}\right\} } \\
& {[1 / / 1,0 / / 1] \quad\left\{(\mathscr{K}, \mathscr{B}): \Phi_{3}, \overline{\mathrm{H}}_{5}\right\} }
\end{aligned}
$$

Как видно из таблищы 1 , из 68 канонически форм, соответствующих 34 горновским функциям, 42 формы относятся к гауссовскому типу (2//1), а 21 форма - к куммеровскому типу (1//1). Это означает, что формулы (19)-(21) содержат в качестве непосредственных частных случаев $42 \cdot 3+21=147$ линейных преобразований горновских рядов. Такие преобразования приводят, как правило, к негорновским функциям высших порядков. В некоторых случаях при наложении дополнительных условий на параметры исходного ряда преобразованные ряды могут быть выражены в виде горновских функций. В этих случаях допустимы повторные линейные преобразования ряда по другому аргументу ряда, что приводит к значительному увеличению полного списка преобразований.

В качестве иллюстративного примера рассмотрим случай горновской функции $H_{1}$. Стандартное условное обозначение [5], полное “инструктивное" обозначение (см. п. I.2) и две канонические формы ряда $H_{1}$ (см. определение 1$)$ имеют вид

$$
\begin{aligned}
H_{1}\left[\alpha, \beta, \gamma, \delta ; x_{1}, x_{2}\right] & ={ }^{2} F\left[\langle\alpha \mid 1, \overline{1}\rangle, \beta: * / / \delta ; \gamma / / * ; x_{1}, x_{2}\right] \\
& =F\left[\begin{array}{cc}
\langle\beta \mid 1,1\rangle,\langle\alpha \mid 1, \overline{1}\rangle: * ; \gamma ; x_{1}, x_{2} \\
\langle\delta \mid 1,0\rangle & : * ; *
\end{array}\right] \\
& =F\left[\begin{array}{cc}
\langle\beta \mid 1,1\rangle,\langle\gamma \mid 0,1\rangle: * ; * ;-x_{1},-x_{2} \\
\langle 1-\alpha \mid \overline{1}, 1\rangle & : \delta ; *
\end{array}\right] .
\end{aligned}
$$


В случае ряда $H_{1}$ любые преобразования вида $L_{i}^{n}($ см. (19)-(21)) приводят к представлению $H_{1}$ в виде негорновских рядов. Частные случаи преобразований $L_{i}^{n}$, приводящие к упрощению этих рядов, имеют вид

$$
\begin{aligned}
H_{1}\left[\alpha, \beta, \gamma, \alpha+\gamma ; x_{1}, x_{2}\right] & =\left(1-x_{1}\right)^{-\beta} F_{4}\left[\gamma, \beta, \alpha+\gamma, 1-\alpha ; \frac{x_{1}}{x_{1}-1}, \frac{x_{2}}{x_{1}-1}\right] \\
& =\left(1+x_{2}\right)^{-\beta} G_{1}\left[\beta, 1-\alpha-\gamma, \alpha ;-\frac{x_{1}}{x_{2}+1},-\frac{x_{2}}{x_{2}+1}\right], \\
H_{1}\left[\alpha, \beta, 1-\alpha, \delta ; x_{1}, x_{2}\right] & =\left(1-x_{1}\right)^{-\beta} F_{4}\left[\delta-\alpha, \beta, \delta, \delta-\alpha ; \frac{x_{1}}{x_{1}-1}, \frac{x_{2}}{x_{1}-1}\right] \\
& =\left(1+x_{2}\right)^{-\beta} H_{3}\left[\beta, \alpha, \delta ; \frac{x_{1} x_{2}}{\left(x_{2}+1\right)^{2}}, \frac{x_{1}}{x_{2}+1}\right] \\
H_{1}\left[\alpha, \beta, \gamma, \beta ; x_{1}, x_{2}\right] & =\left(1-x_{1}\right)^{-\alpha} F_{4}\left[\beta, \gamma, \beta, 1-\alpha ; x_{1} x_{2}, x_{1} x_{2}-x_{2}\right] .
\end{aligned}
$$

Отметим, что формулы $(22),(24)$ следуют из преобразования $L_{1}^{1}$ и замечания $4 \mathrm{a},(23)-$ из $L_{2}^{2}$ и замечания $4 \mathrm{a},(25)$ - из $L_{2}^{2}$ и предложения $1,(26)$ - из $L_{2}^{1}$ и предложения 1 . Насколько известно автору, ни одна из этих формул в литературе ранее не была отмечена.

В связи с преобразованиями аппелевых рядов $F_{1}, F_{2}$ в ряды того же типа Эрдейи отмечает (см. [5, п. 5.11]), что "ни для одного из других полных гипергеометрических рядов от двух переменных не известно преобразований такого типа”.

Укажем в связи с этим замечанием формулу

$$
\begin{aligned}
& H_{1}\left[\alpha, \beta, \gamma, \beta ; x_{1}, x_{2}\right]=\left(1-x_{1}\right)^{-\alpha}\left(1+x_{2}-x_{1} x_{2}\right)^{-\gamma} \\
& \quad \times H_{1}\left[1-\alpha-\beta, \gamma, \alpha+\beta, 1-\alpha ; \frac{x_{2}\left(x_{1}-1\right)}{\left(x_{1} x_{2}-x_{2}-1\right)}, \frac{x_{1} x_{2}}{x_{1} x_{2}-x_{2}-1}\right],
\end{aligned}
$$

которая следует из определения ряда $F_{4}$ и очевидных замен переменных и параметров, приводящих правые части $(24),(26)$ к одинаковому виду.

В связи с этим же замечанием отметим, что ряд $H_{2}[\alpha, \beta, \gamma, \delta, \varepsilon]$ [5] в отличие от $H_{1}$ (cp. с (27)) допускает преобразование в ряд того же типа при произвольных значения параметров. В самом деле, применяя к ряду преобразование $L_{2}^{1}$, получаем искомое соотношение в виде

$$
H_{2}\left[\alpha, \beta, \gamma, \delta, \varepsilon ; x_{1}, x_{2}\right]=\left(1-x_{1}\right)^{-\alpha} H_{2}\left[\alpha, \varepsilon-\beta, \gamma, \delta, \varepsilon ; \frac{x_{1}}{x_{1}-1}, \frac{x_{2}}{1-x_{1}}\right] .
$$

Таким образом, полученные в п. 5 преобразования позволяют уточнить замечание Эрдейи, пополняя список рядов $F_{1}, F_{2}$ еще двумя рядами.

За исключением формулы (28) все остальные линейные преобразования ряда $H_{2}$ приводят к негорновским рядам высших (третьего и четвертого) порядков. Упрощая эти ряды за счет подбора специальньх значений параметров, получаем преобразования

$$
\begin{aligned}
H_{2}\left[\alpha, \beta, \gamma, \varepsilon-\alpha, \varepsilon ; x_{1}, x_{2}\right] & =\left(1-x_{1}\right)^{-\beta} F_{2}\left[\varepsilon-\alpha, \beta, \gamma, \varepsilon, 1-\alpha ; \frac{x_{1}}{x_{1}-1},-x_{2}\right] \\
& =\left(1+x_{2}\right)^{-\gamma} G_{2}\left[\beta, \gamma, 1-\varepsilon, \alpha ;-x_{1},-\frac{x_{2}}{1+x_{2}}\right], \\
H_{2}\left[\alpha, \beta, \gamma, 1-\alpha, \varepsilon ; x_{1}, x_{2}\right] & =\left(1-x_{1}\right)^{-\beta} F_{2}\left[\varepsilon-\alpha, \beta, \gamma, \varepsilon, \varepsilon-\alpha ; \frac{x_{1}}{x_{1}-1},-x_{2}\right] \\
& =\left(1+x_{2}\right)^{-\gamma} F_{1}\left[\beta, \alpha, \gamma, \varepsilon ; x_{1}, \frac{x_{1} x_{2}}{1+x_{2}}\right]
\end{aligned}
$$


не вьводящие из класса горновских функций. Формулы $(29),(31)$ следуют из преобразования $L_{1}^{1}$ и замечания $4 \mathrm{a},(30)$ - из $L_{2}^{2}$ и того же замечания, $(32)$ - из $L_{2}^{2}$ и предложения 1. Из этих четырех соотношений формулы (29), (30) принадлежат Эрдейи [6], а две другие, по-видимому, отсутствуют в литературе.

Простота и систематичность приемов, используемых для анализа свойств гипергеометрических рядов с помощью соотношений (18)-(21), указывают на особую перспективность применения этих соотношений для автоматизации вывода формул преобразования и правил редукции гипергеометрических рядов с помощью программы для ЭВМ. С помощью предварительной версии такой программы, упомянутой во введении, были успешно осуществлены на ЭВМ все приведенные вьше преобразования горновских функций. Значительно более сложные примеры применения этой программы для вывода формул приведения функций Гельфанда, связанных с грассманианами $G_{2,4}$ и $G_{3,6}$, даны в [7].

ЗАМЕЧАНИЯ ПРИ КОРРЕКТУРЕ. За время, прошедшее после представления статьи в редакцию "Математических заметок", были опубликованы работы [8]-[15], имеющие более или менее близкое отношение к материалу данной заметки. Применение общих линейных преобразований к $\Omega$-сомножителям, входящим в факторизованные представления функции Аппеля $F_{4}$ и функций Горна $H_{1}$ и $G_{2}$, позволило получить для этих трех функций специальные дробно-линейные преобразования [8], не выполняющиеся ни для каких других гипергеометрических рядов. Метод факторизации был применен для вывода некоторых новых формул сложения для гипергеометрических функций [9]; в той же работе дан частньй пример применения общего линейного преобразования куммеровского типа. Совместное применение общих и специальных линейньх преобразований позволило осуществить с помощью программы символических преобразований [10] компьютерную генерацию 36 гипергеометрических представлений для функции Апшеля $F_{4}\left[a_{1}, a_{2}, a_{1}, b_{2} ; x, y\right][11]$. Сформулированные в настоящей заметке элементарные правила редукции позволили сформулировать эффективньй алгоритм поиска нетривиальных формул приведения гипергеометрических рядов от нескольких переменных [12], [7]. Возможность компютерной реализации алгоритмов, полученных с помощью метода факторизации, указьвает на перспективность разработки с помощью Internet - технологии математического сервера, основным содержанием которого служила бы не база данных (как, например, в американском проекте [13]), а база знаний, использующая в качестве команд "языка надстройки” как собственно операции метода факторизации, так и базисные соотношения между гипергеометрическими рядами, полученные с помощью этого метода [14]. Сравнение стандартных методов с методом факторизации, выявляющее характерные особенности применения последнего на примере обобщения классического соотношения между ассоциированными функциями Бесселя, дано в [15].

\section{СПИСОК ЦИТИРОВАННОЙ ЛИТЕРАТУРЫ}

[1] Ниукканен А. В. Распространение принципа факторизации на гипергеометрические ряды общего вида // Матем. заметки. 2000. Т. 67. №4. С. 573-581.

[2] Niukkanen A. W. Generalized operator reduction formulae for multiple hypergeometric series ${ }^{N} F\left(x_{1}, \ldots, x_{N}\right)$ // J. Phys. A. Math. Gen. 1984. V. 17. P. L731-L736.

[3] Ниукканен А. В. Новый метод в теории гипергеометрических рядов и специальных функций математической физики // УМН. 1988. Т. 43. № 3. С. 191-192.

[4] Ниукканен А. В. Новый подход к теории гипергеометрических рядов и специальных функций математической физики // Матем. заметки. 1991. Т. 50. № 1. С. 65-73. 
[5] Бейтмен Г., Эрдейи А. Высшие трансцендентные функции. Т. І. М.: Наука, 1973.

[6] Erdelyi A. Hypergeometric functions of two variables // Acta Math. 1950. V. 83. P. 131-164.

[7] Ниукканен А. В., Парамонова О. С. Линейные преобразования и формулы приведения гипергеометрических функций Гельфанда, связанных с грассманианами $G_{2,4}$ и $G_{3,6} / /$ Матем. заметки. (в печати).

[8] Ниукканен А. В. Метод факторизации и особые преобразования функции Аппеля $F_{4}$ и функций Горна $H_{1}$ и $G_{2} / /$ УМН. 1999. Т. 54. №6. С. 169-170.

[9] Niukkanen A. W. Operator factorization method and addition formulas for hypergeometric functions // International J. Integral Transforms Special Functions. 2001. V. 11. P. 25-48.

[10] Парамонова О. С., Ниукканен А. В. Аналитические преобразования гипергеометрических рядов с помощью новой надстройки над системой Maple // Программирование. 1998. № 6. C. $25-26$.

[11] Парамонова О. С., Ниукканен А. В. Компьютерный анализ формул преобразования функций Аппеля и Горна // Программирование. (в печати).

[12] Парамонова О.С., Ниукканен А.В. Новые алгоритмы поиска формул приведения гипергеометрических рядов от нескольких переменных // Программирование. 2000. №1. C. $62-63$.

[13] Digital Library of Mathematical Functions. Homepage at National Institute of Standards and Technology: http://dlmf .nist.gov/.

[14] Mitrofanov V. A., Paramonova O. S., Niukkanen A. W. A project of remote hypergeometric calculator based on the use of operator factorization method and internet client-server tecnology // Proceedings of 6th International IMACS Conference on Applications of Computer Algebra. St.-Petersburg, 2000. P. 107-109.

[15] Niukkanen A. W., Perevozchikov I. V., Lurie V. A. A generalization of a classical relation between $J_{\nu+n}(z), J_{\nu}(z)$ and $J_{\nu-1}(z)$ with comments on the modern state and trends in the theory of special functions // Fractional Calculus Appl. Anal. 2000. V. 3. № 2. P. 119-132.

Институт геохимии и аналитической химии

Поступило

им. В. И. Вернадского РАН

08.10 .1998

Исправленный вариант

08.07.2001 